\title{
Genome wide characterization of simple sequence repeats in watermelon genome and their application in comparative mapping and genetic diversity analysis
}

Huayu Zhu ${ }^{1 \dagger}$, Pengyao Song ${ }^{1 \dagger}$, Dal-Hoe Koo ${ }^{2}$, Luqin Guo ${ }^{1}$, Yanman Li ${ }^{1}$, Shouru Sun ${ }^{1}$, Yiqun Weng ${ }^{2,3^{*}}$ and Luming Yang ${ }^{1 *}$ (D)

\begin{abstract}
Background: Microsatellite markers are one of the most informative and versatile DNA-based markers used in plant genetic research, but their development has traditionally been difficult and costly. The whole genome sequencing with next-generation sequencing (NGS) technologies provides large amounts of sequence data to develop numerous microsatellite markers at whole genome scale. SSR markers have great advantage in cross-species comparisons and allow investigation of karyotype and genome evolution through highly efficient computation approaches such as in silico PCR. Here we described genome wide development and characterization of SSR markers in the watermelon (Citrullus lanatus) genome, which were then use in comparative analysis with two other important crop species in the Cucurbitaceae family: cucumber (Cucumis sativus L.) and melon (Cucumis melo L.). We further applied these markers in evaluating the genetic diversity and population structure in watermelon germplasm collections.

Results: A total of 39,523 microsatellite loci were identified from the watermelon draft genome with an overall density of 111 SSRs/Mbp, and 32,869 SSR primers were designed with suitable flanking sequences. The dinucleotide SSRs were the most common type representing $34.09 \%$ of the total SSR loci and the AT-rich motifs were the most abundant in all nucleotide repeat types. In silico PCR analysis identified 832 and 925 SSR markers with each having a single amplicon in the cucumber and melon draft genome, respectively. Comparative analysis with these crossspecies SSR markers revealed complicated mosaic patterns of syntenic blocks among the genomes of three species. In addition, genetic diversity analysis of 134 watermelon accessions with 32 highly informative SSR loci placed these lines into two groups with all accessions of C.lanatus var. citorides and three accessions of $C$. colocynthis clustered in one group and all accessions of C. lanatus var. lanatus and the remaining accessions of $C$. colocynthis clustered in another group. Furthermore, structure analysis was consistent with the dendrogram indicating the 134 watermelon accessions were classified into two populations.

(Continued on next page)
\end{abstract}

\footnotetext{
*Correspondence: weng4@wisc.edu; ylmhort@163.com

${ }^{\dagger}$ Equal contributors

${ }^{2}$ Horticulture Department, University of Wisconsin, Madison, WI 53706, USA

${ }^{1}$ College of Horticulture, Henan Agricultural University, 63 Nongye Road,

Zhengzhou 450002, China

Full list of author information is available at the end of the article
} 
(Continued from previous page)

Conclusion: The large number of genome wide SSR markers developed herein from the watermelon genome provides a valuable resource for genetic map construction, QTL exploration, map-based gene cloning and marker-assisted selection in watermelon which has a very narrow genetic base and extremely low polymorphism among cultivated lines. Furthermore, the cross-species transferable SSR markers identified herein should also have practical uses in many applications in species of Cucurbitaceae family whose whole genome sequences are not yet available.

Keywords: SSR, Watermelon, Comparative genomics, Synteny, Cucurbits, Genetic diversity

\section{Background}

Watermelon (Citrullus lanatus) is an important horticultural crop and one of the most consumed fresh fruits globally. It belongs to the genus Citrullus, which contains four diploid species: Citrullus lanatus (Thunb.) Mat-sum. \& Nakai, C. colocynthis (L.) Schrad, C. ecir rhosus Cogn. and C. rehmii De Winter [1, 2]. Among these four species, Citrullus lanatus includes the cultivated watermelon (C. lanatus var. lanatus) which thrives in West Africa and has been cultivated widely worldwide (also called 'egusi' melon) and the preserving melon (C. lanatus var. citroides) that is grown in Southern Africa (also called 'tsamma' melon) $[3,4]$, and C. colocynthi ('bitter apple') is a perennial species grown in sandy areas throughout northern Africa, south-western Asia, and the Mediterranean [2, 5]. The long term domestication and selection for desirable horticultural qualities has made the cultivated watermelon with a narrow genetic base and susceptibility to a large number of diseases and pests [6]. Evaluating the phylogenetic relationships among different species in Citrullus genus will help us for improving watermelon cultivars in diseases resistance [1]. Watermelon has a small genome of $425 \mathrm{Mb}$, and the genome of the elite Chinese watermelon line 97103 [7] and the American heirloom watermelon cultivar Charleston Gray have been sequenced and released in cucurbit genomics database (www.icugi.org). The availability of these genomic resources of watermelon have greatly promoted the fundamental researches including the development of molecular markers and genetic map construction $[8,9]$, gene/QTL mapping $[10,11]$, molecular breeding, and comparative genomics [12].

Microsatellites or simple sequence repeats (SSRs), are one of the most commonly used marker in many genetic applications since the early 1990s including mapping, fingerprinting, genetic diversity and population structure analysis [13-16]. Because of their reproducibility, multiallelism, co-dominance, relative abundance, good genome coverage and versatile platforms to genotype, the use of microsatellites is likely to continue to be used for some years to come. Furthermore, they are comparatively cheap to genotype and provide more population genetic information per marker than bi-allelic markers such as single nucleotide polymorphisms (SNP) [17, 18]. A single set of microsatellite markers can be used to genotype several related species, but SNP markers in general lack crossspecies utility, and are therefore only suitable for population and paternity studies in a single species [19-21]. The microsatellite loci can be detected both in genomic sequences and expressed sequence tag (EST), which were named genomic SSRs and EST-SSR. EST-SSRs are useful for genetic analysis, but their relatively low polymorphism and the high possibility of no gene-rich regions in the genome are limitations to their use. In contrast, genomic SSRs are highly polymorphic and tend to be widely distributed throughout the genome, resulting in better map coverage [22].

With the rapid development of sequencing technologies, whole-genome sequences (WGS) are becoming increasingly available. These DNA sequences are valuable resources for SSR development and genome wide identification of SSR have been investigated in many plant species, such as cucumber [23], foxtail millet [24] and Brassica [25]. Together with the advantage of in silico analysis, this approach has the potential to develop highly polymorphic SSR markers to suit various applications such as comparative studies in species where limited or no sequence information is available [12, 26, 27]. However, large scale development of microsatellite markers was not realized until the whole genome sequence of watermelon was available [7]. Recently Ren et al. [8] identified 13,744 putative SSR loci and 1877 unique SSRs with long repeat motifs were selected for polymorphism analysis and genetic map construction. The usefulness of these watermelon microsatellite markers has already been demonstrated in recent linkage mapping [11] and genetic diversity studies [28]. Despite such progresses, the number of robust, informative and userfriendly markers publicly available for watermelon is still insufficient for some applications, particularly considering the low intra-specific polymorphism level of microsatellite markers in watermelon. The availability of microsatellite markers distributed throughout the genome would facilitate the development of high resolution maps or rapid saturation of target genomic regions, which is instrumental for applications like positional gene cloning and detailed 
comparative mapping. Such molecular resources would benefit the watermelon research and breeding community.

Watermelon $(2 n=2 x=22)$ belongs to the Cucurbitaceae family which includes several economically important species, such as melon (Cucumis melo, $2 \mathrm{n}=2 \mathrm{x}=24$ ) and cucumber (Cucumis sativus L., $2 \mathrm{n}=2 \mathrm{x}=14$ ). Draft genome assemblies for the three species are now publicly available $[23,27,29,30]$ and the availability of large numbers of molecular markers has made it possible to define more clearly syntenic relationships among them. To access the nature of evolutionary events leading to modern cucurbit genome structures, Huang et al. [29] established the syntenic relationship between cucumber and watermelon by alignment of 136 watermelon marker sequences mapped from watermelon linkage groups in 9930 cucumber draft genome and Guo et al. [7] investigated the chromosometo-chromosome relationships within the Cucurbitaceae family by comparative mapping and identified the complicated syntenic patterns illustrated as mosaic chromosome-to-chromosome orthologous relationships among watermelon, cucumber and melon. In the genus Cucumis genus, syntenic relationships among chromosomes of cucumber, melon and C. hystrix have been extensively analysed [12, 27, 30, 31]. For example, 91 syntenic blocks were divided between cucumber and melon, and 53 syntenic blocks were identified betweeen cucumber and $C$. hystrix by comparative mapping and comparative fluorescence in situ hybridization (FISH). These findings revealed a high degree of complexity of structure rearrangements after cucumber and melon diverging from their common ancestor. However, the syntenic relationship and chromosomal rearrangements between watermelon with cucumber and melon are still largely fragmented and incomplete.

In this study, we identified genome wide SSR in watermelon and characterized the distribution and frequency of different motifs and repeats. We further identified cross-species transferable SSR markers in cucumber and melon by in silico PCR analysis, and established syntenic relationships between watermelon and cucumber, as well as between watermelon and melon chromosomes based on shared SSR markers. In addition, 32 highly informative SSR markers were identified and used to evaluate the genetic diversity and population structure of 134 Citrullus accessions including C. lanatus var. lanthus, $C$. lanatus var. citroides and C. colocynthis.

\section{Results}

The frequency and distribution of different SSR types in watermelon genome

A total of 39,523 microsatellite sequences were identified in the released $353.5 \mathrm{Mb}$ genomic sequences of East Asia watermelon cultivar 97103 with more conserved criteria than that in cucumber [23]. The total length of all SSR sequences was estimated to be $0.28 \%$ of the draft genome assembly with an average of 111 SSR/Mb. Among different nucleotide types, the microsatellite frequency was negatively correlated with the number of nucleotide. For example, dinucleotide repeats were the most abundant accounting for $34.09 \%$ of the total SSR loci discovered, followed by tri- $(22.64 \%)$ and tetra$(13.83 \%)$, and octonucleotides were the least frequent repeat types $(3.27 \%)$ (Table 1$)$. We also investigated the SSR motif distribution with regard to repeat number. For all seven SSR types, microsatellite frequency decreased as the number of repeat units increased, which was more obvious with longer SSR motifs (Fig. 1). As a consequence, the mean repeat number in dinucleotides (12.29) was about four times the number of hepta- and octonucleotide (3.19 and 3.14 respectively) (Table 1).

We examined the nucleotide composition of each motif type and found that some combinations of nucleotides were more prevalent than others in each class. For example, the AT motif was dramatically overrepresented in dinucleotide motifs, and it was also the most frequent motif in the entire watermelon genome, which accounting for $25.07 \%$ of the total SSR loci discovered. Similarly, the AAT, AAAT, AAAAT, AAAAAT, AAAAAAT and AAAAAAAT were the most abundant repeats types in each class (Additional file 1: Figure S1). We further investigated the frequency and distribution of different SSR types in each watermelon chromosome. The frequency of microsatellite loci was not correlated with the chromosome size (Fig. 2). For example, chromosome 6 had the highest density of $129.03 \mathrm{SSR} / \mathrm{Mb}$, while it was one of the smallest chromosomes of watermelon (Table 2). The largest number of microsatellite was detected on chromosome 5 (4349), followed by chromosome 1 (4264) and 2 (3980), and the least SSR number was located on chromosome 4 (2456). There were even 889 SSR loci detected on these scaffolds not yet anchored to any of the 11 chromosomes (designated as chr0) with a very low density of $36 \mathrm{SSR} / \mathrm{Mb}$.

The genomics DNA sequences containing these microsatellites were screened for PCR primer design using Primer3, and 33,810 SSR microsatellite loci contained suitable flanking sites for SSR primer design. Finally, we designed 32,869 SSR primers with some SSR loci included in the same primers as compound SSRs. The exact positions of these SSRs in the watermelon chromosomes, as well as information on repeat motifs and expected PCR product length are presented in Additional file 2: Table S2.

\section{Comparative analysis of watermelon SSR markers in cucumber and melon genome}

To identify genome wide cross-species watermelon SSR markers, all 32,869 SSR markers were used for 
Table 1 The distribution of different nucleotide repeats in watermelon genome

\begin{tabular}{lllllc}
\hline Nucleotide & $\begin{array}{l}\text { Number } \\
\text { of loci } \\
\text { identified }\end{array}$ & $\begin{array}{l}\text { Frequency } \\
(\%)\end{array}$ & $\begin{array}{l}\text { Mean } \\
\text { repeat } \\
\text { number }\end{array}$ & $\begin{array}{l}\text { Number } \\
\text { of loci } \\
\text { primer } \\
\text { designed }\end{array}$ & $\begin{array}{l}\text { Percentage } \\
\text { SSRs suitable } \\
\text { for primer } \\
\text { design (\%) }\end{array}$ \\
\hline Di & 13474 & 34.09 & 12.29 & 11353 & 84.26 \\
Tri & 8947 & 22.64 & 10.56 & 7718 & 86.26 \\
Tetra & 5465 & 13.83 & 5.72 & 4552 & 83.29 \\
Penta & 4205 & 10.64 & 4.43 & 3681 & 87.54 \\
Hexa & 2082 & 5.28 & 4.43 & 1938 & 93.08 \\
Hepta & 4059 & 10.27 & 3.19 & 3412 & 84.06 \\
Octo & 1291 & 3.27 & 3.14 & 1156 & 89.54 \\
Total & 39523 & 100 & & 33810 & 85.55 \\
\hline
\end{tabular}

in silico PCR analysis using cucumber and melon draft genome sequences as the templates. We identified $832(2.53 \%)$ SSR markers with a single in silico PCR product in the seven pseudochromosomes of cucumber Gy14, whereas 59 SSR markers had at least two products and CISSR20036 had the largest number of 25 products which were distributed on all seven cucumber chromosomes. These cross-species SSR markers covered $190.42 \mathrm{Mb}$ accounting for $98.87 \%$ of the cucumber assembly. The physical positions of common markers between watermelon and cucumber are presented in Additional file 2: Table S3. The number of cross-species SSR markers in melon genome was comparable to that in cucumber. Totally, $925(2.81 \%)$ of all SSR markers had one in silico PCR products in melon genome assembly, and 44 SSR markers had at least two products. The cross-species SSR markers in melon were spanned 310.94 Mb accounting for $98.29 \%$ of melon genome assembly. The physical positions of cross-species markers between melon and watermelon are presented in Additional file 2: Table S4.

The distribution and frequency of cross-species SSR markers on each chromosome in cucumber and melon were also investigated. In cucumber, there was an average of 119 common SSR markers on each chromosome with a density of $4.32 \mathrm{SSR} / \mathrm{Mb}$. Chromosome C3 had the largest number of 183 common markers and C7 had the least number of 68 common markers, which was largely consistent with the physical lengths of the two chromosomes. In melon, of these 925 cross-species SSR markers, 63 were located in the unassembled chromosome, so the remaining 862 common SSR markers were mapped in 12 chromosomes with an average of 72 SSR markers on each chromosome (Additional file 2: Table S4). Melon chromosome VI had the largest number of 98 common markers and the highest density of 3.31SSR/ $\mathrm{Mb}$. Though the chromosome $\mathrm{X}$ had the least number of 45 common markers, the chromosome $\mathrm{V}$ had the lowest density of $2.0 \mathrm{SSR} / \mathrm{Mb}$. There was no direct correlation between chromosome size and number of cross-species SSR markers. This is probably more dependent on the conservation of syntenic regions between species in particular chromosomes.

By comparing the two cross-species SSR marker sets in cucumber and melon, 448 SSR markers were further identified shared among all three genomes (Additional file 2: Table S5). Within each chromosome, fewer markers were found around the centromeres in watermelon; most of the common SSR markers were distally distributed on each chromosome (Fig. 3). The expected in silico PCR products of 448 SSR markers in watermelon genome were also used to BLAST search in EST, unigenesand CDS

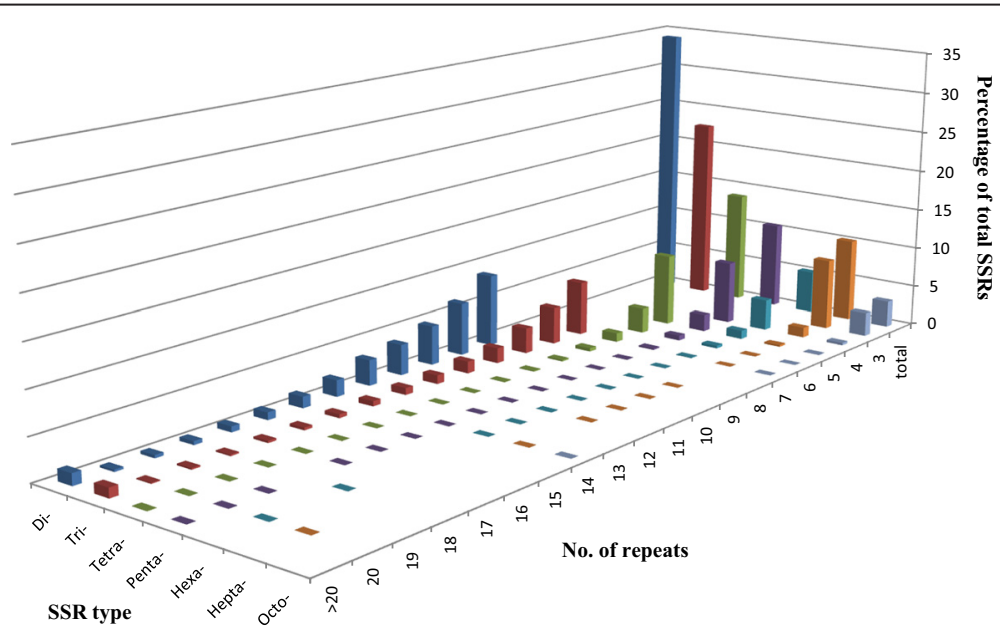

Fig. 1 Distribution of SSR motif repeat numbers and relative frequency in watermelon genome. The vertical axis shows the abundance of microsatellites that have different motif repeat numbers (from 3 to $>20$ ), which are discriminated by legends of different colours 


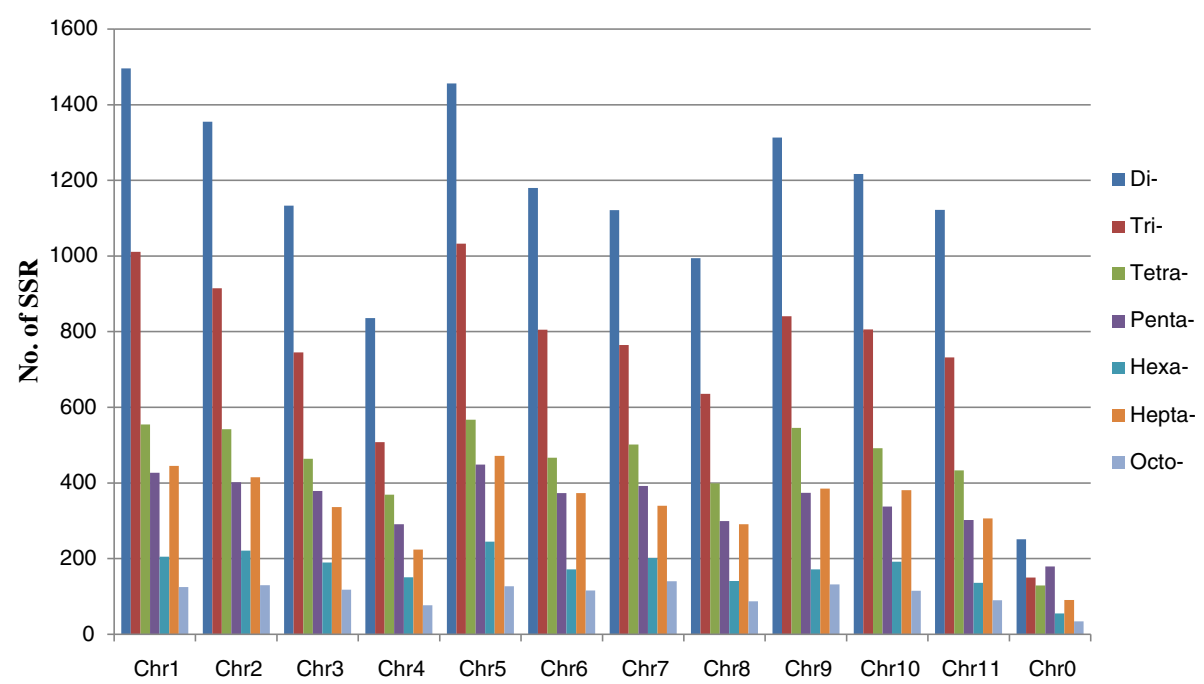

Fig. 2 The distribution of SSR repeat types on each chromosome in watermelon. The vertical axis shows the number of microsatellites from dinucleotide to octonucleotide which are discriminated by different colours. The horizontal axis show different chromosomes of watermelon and chro represent all the chromosomal unanchored scaffolds

database of watermelon, respectively. Of these, 21 SSR markers were expressed in EST database, 19 SSR markers were expressed in unigene database, and 210 of them were located in the coding regions suggesting these SSR markers are related to gene function (Additional file 2: Table S5). For different nucleotides repeats contained in the 448 SSR markers, the dinucleotides had the highest frequency of $87(34.42 \%)$, followed by tri- 64 (27.27\%) and hepta- 20 (14.29\%). The frequency of different motifs was further compared in the same nucleotides repeat. Among the dinucleotides, AG/CT had the most abundant with a frequency of $59.12 \%$, followed by AT/TA (33.33 \%) and AC/GT (7.55 \%), while AAG/CTT was the most abundant in trinucleotides with a frequency of $50.79 \%$.
Chromosome synteny of watermelon with cucumber and melon

Of these 832 cross-species SSR markers between watermelon and cucumber, 11 were located on chr0 of watemelon. Based on their chromosomal positions of the remaining 821 SSR markers in both genomes, syntenic relationships between watermelon and cucumber chromosomes could be directly inferred and visualized in Fig. 3A. The main syntenic chromosomes between watermelon and cucumber revealed complex patterns for different chromosomes, and the main syntenic relationships could be listed in Table 3 . Watermelon chromosome W3 and W10 had the simplest syntenic pattern with cucumber, and each of them was mainly syntenic to two cucumber chromosomes. For example, W3 had 55 shared SSR markers

Table 2 The distribution of nucleotide repeats on different chromosomes

\begin{tabular}{|c|c|c|c|c|c|c|c|c|c|c|c|}
\hline Nucleotide & Chr1 & Chr2 & Chr3 & Chr4 & Chr5 & Chr6 & Chr7 & Chr8 & Chr9 & Chr10 & Chr11 \\
\hline $\mathrm{Di}-$ & 1496 & 1355 & 1133 & 836 & 1456 & 1180 & 1121 & 994 & 1313 & 1217 & 1122 \\
\hline Tri- & 1011 & 915 & 745 & 508 & 1033 & 805 & 765 & 636 & 841 & 806 & 732 \\
\hline Tetra- & 555 & 542 & 464 & 369 & 567 & 467 & 502 & 399 & 546 & 492 & 433 \\
\hline Penta- & 427 & 402 & 379 & 291 & 449 & 373 & 392 & 299 & 374 & 338 & 302 \\
\hline Hexa- & 205 & 221 & 190 & 151 & 245 & 172 & 202 & 141 & 172 & 192 & 136 \\
\hline Hepta- & 445 & 415 & 336 & 224 & 472 & 373 & 340 & 291 & 385 & 381 & 306 \\
\hline Octo- & 125 & 130 & 118 & 77 & 127 & 116 & 140 & 87 & 132 & 115 & 90 \\
\hline Total & 4264 & 3980 & 3365 & 2456 & 4349 & 3486 & 3462 & 2847 & 3763 & 3541 & 3121 \\
\hline Chr. Size (Mb) & 34.08 & 34.41 & 28.94 & 24.32 & 33.72 & 27.02 & 31.48 & 26.15 & 34.99 & 28.42 & 27.11 \\
\hline Density (SSRs/Mb) & 125.11 & 115.65 & 116.28 & 101.00 & 128.99 & 129.03 & 109.98 & 108.88 & 107.55 & 124.60 & 115.14 \\
\hline
\end{tabular}



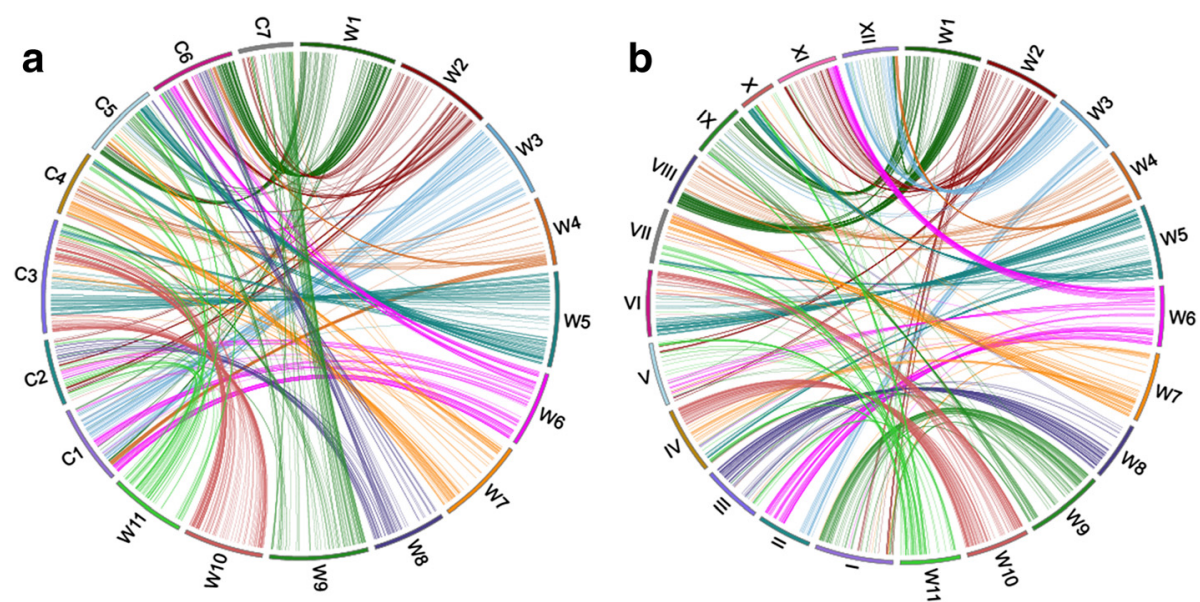

Fig. 3 Syntenic relationships of watermelon with cucumber (a) and melon (b) chromosomes. Chromosome synteny between watermelon and cucumber is based on 821 cross-species markers (A); synteny between watermelon and melon is based on 850 cross-species markers. W1-W11 represent watermelon eleven chromosomes, C1-C7 represent cucumber seven chromosomes and I-XII represent melon twelve chromsomes. Syntenic blocks are connected by with the same colour lines from watermelon chromosomes

with cucumber, 52 of them were located on $\mathrm{C} 1$ and the remaining three SSR markers (ClSSR08129, ClSSR08186 and CISSR08224) were located on C5 (Fig. 3A and Additional file 2: Table S3). Watermelon chromosome W2 and W11 showed the most complicated patterns and each of them were syntenic to five cucumber chromosomes. Chromosome W9 was syntenic to four cucumber chromosomes and the remaining six watermelon chromosomes had similar syntenic pattern with each of them corresponding to three cucumber chromosomes. According to the cross-species SSR markers sharing continuous physical positions on both genomes, the 11 watermelon

Table 3 Major syntenic chromosomes among watermelon, cucumber and melon

\begin{tabular}{|c|c|c|}
\hline Watermelon & Cucumber & Melon \\
\hline W1 & $\mathrm{C} 1(14), \mathrm{C5}(35), \mathrm{C} 6(60)$ & VIII(69), IX(31), XII(20) \\
\hline W2 & $\begin{array}{l}C 2(26), C 4(3), C 5(4), C 6(22), \\
C 7(15)\end{array}$ & $I(17), V(18), X(3), X \mid(42)$ \\
\hline W3 & $\mathrm{C1}(52), \mathrm{C} 5(3)$ & $\|(15), I X(4), X\|(38)$ \\
\hline W4 & C1(19), C4(17), C6(10) & VIII(23), XII(15) \\
\hline W5 & C3(67), C4(13), C5(33) & IV(13), VI(58), VII(11), X(28) \\
\hline W6 & C1 (45), C2(11), C6(29) & $\|(41), V(12), X \mid(35)$ \\
\hline W7 & C3(12), C4(28), C5(13) & I(5), IV(14), VII(32) \\
\hline W8 & C2(33), C5(4), C6(28) & $\|||(57)$ \\
\hline W9 & C3(9), C5(11), C6(4), C7(48) & I(44), IV(6), IX(14), V(3) \\
\hline W10 & C3(86), C4(5) & IV(47), VI(35), VII(4) \\
\hline W11 & $\begin{array}{l}\text { C2(11), C3(8), C4(13), C5(12), } \\
\text { C6(6) }\end{array}$ & $\begin{array}{l}\text { III(7), IV(6), V(19), VII(17), IX(5), } \\
X(11)\end{array}$ \\
\hline
\end{tabular}

The number in the bracket means the shared SSR markers on these chromosomes in melon and cucumber. The syntenic chromosomes with less than three shared markerswere not listed here chromosomes were further divided into different syntenic blocks with each block containing at least three SSR markers. 84 syntenic blocks could be recognized, 44 of which were collinear, and the remaining 40 blocks showed inversions between watermelon and cucumber genomes (Additional file 2: Table S3). Each watermelon chromosome contained 5-11 discrete syntenic blocks. The largest block was WCB73 in chromosome $\mathrm{W} 10$ that spanned $7.70 \mathrm{Mb}$ in cucumber chromosome C3. The watermelon syntenic block WCB6 had the largest number (55) of shared SSR markers which were collinear between watermelon W1 and cucumber C6.

Similar comparison was carried out using the crossspecies SSR markers between watermelon and melon. Of the 925 cross-species SSR markers between the two genomes, 850 had unambiguous chromosome locations which were used to infer the syntenic relationships between this two species (Fig. 3B and Additional file 2: Table S4). Despite of the similar chromosome numbers in melon and watermelon, the chromosome synteny between them was rather complicated (Additional file 2: Table S4) which was consistent with their far-away phylogenetic distance. In most cases, each watermelon chromosome was syntenic to three melon chromosomes. Watermelon chromosome W11 had the most complicated syntenic pattern which was composed of blocks corresponding to seven melon chromosomes, while W8 showed the simplest pattern which was only syntenic to two melon chromosomes. The whole chromosome W8 was almost syntenic to melon III except for the topmost with two SSR markers (CISSR21281 and ClSSR21359) that were syntenic to melon IX, but from a closer look, 2 and 3 of the 5 blocks (WMB51-55) in W8 were collinear and inverted to 
melon chromosome III, respectively (Additional file 2: Table S4). Among the 81 syntenic blocks assigned, 48 were collinear between the two genomes. For example, 43 shared SSR markers in block WMB70 on the end of watermelon W10 were completely collinear with melon chromosome IV except one marker ClSSR29104 which located on chr0, and this block covered 8.96 and $7.35 \mathrm{Mb}$ in watermelon and melon, respectively (Additional file 2: Table S4). Furthermore, we identified 25 syntenic blocks shared in three genomes which were distributed on 10 watermelon chromosomes (Additional file 2: Table S6), indicating these genomic regions are highly conserved during chromosome evolution.

Previous studies have revealed that watermelon was diverged from the lineage leading to melon and cucumber in the Cucurbitaceae family approximately 20 million years ago [32, 33]. To better understand chromosome evolution in cucurbit species, the watermelon based syntenic block view of cucumber and melon chromosomes were developed in Fig. 4. The arrangement of watermelon syntenic blocks across seven cucumber chromosomes and twelve melon chromosomes indicated complicated mosaic patterns of chromosome evolution in species of the Cucurbitaceae family. In the melon genome, chromosomes II, III, VIII and XI were syntenic to two watermelon chromosomes, while the remaining eight chromosomes contained syntenic blocks corresponding to more than three watermelon chromosomes (Fig. 4). Compared with melon, the syntenic blocks in cucumber were even more complicated with each cucumber chromosome containing syntenic blocks from more than two watermelon chromosomes (Fig. 4). For example, cucumber C7 was largely syntenic to watermelon W2 and W9, while C6 was composed of segment regions from seven watermelon chromosomes.

We verified the SSR-based syntenic relationships among the three species using fluorescence in situ hybridization (FISH) and cucumber C7 as an example. 14 cucumber fosmid probes located on $\mathrm{C} 7$ were selected in this study (Additional file 2: Table S7), where these fosmid probes have been used to confirm the synteny in different Cucumis species in our previous studies [12, 27]. All 14 fosmid probes detected single hybridization signal in watermelon, while they were located on two different chromosomes W2 and W9. Five probes (71.1-71.2, 71.471.6) from short arm and one fosmid (71.8) from long arm of cucumber C7 were located on watermelon chromosome W2, and the remaining eight probes were detected on chromosome W9 (Fig. 5). Two inversions were detected between watermelon W2 and cucumber C7 which were in accordance with block WCB8 and WCB9, indicating this was completely consistent with the results from in silico comparative mapping. The large inversion block WCB62 between the top of watermelon W9 and the end of cucumber $\mathrm{C} 7$ spanned almost $8 \mathrm{Mb}$ in watermelon, which was also confirmed by FISH mapping using probes 71.9-72.4. Compared with cucumber, six probes on

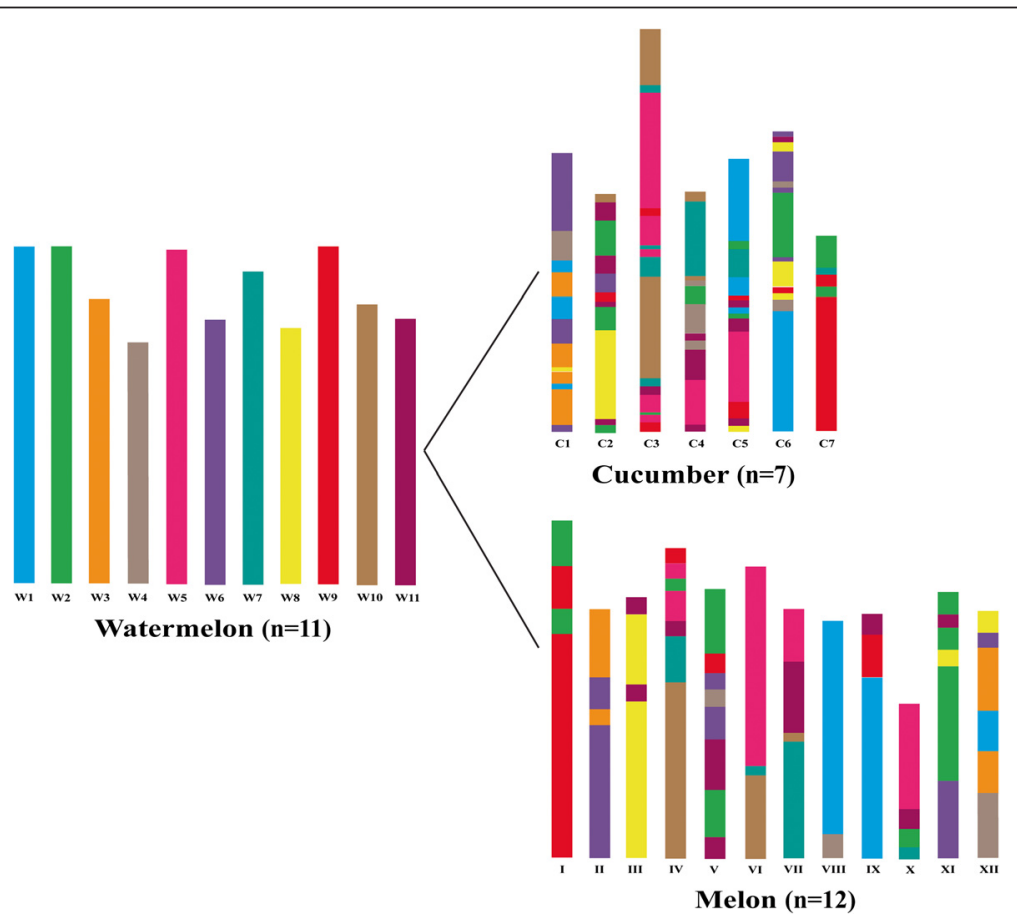

Fig. 4 A syntenic block view of cucumber and melon chromosomes composted of watermelon chromosomes. The different colours represent the eleven chromosomes of watermelon. The mosaic colour pattern of cucumber and melon chromosomes indicated seven cucumber chromosomes and twelve melon chromosomes composed of syntenic blocks from different watermelon chromosomes 


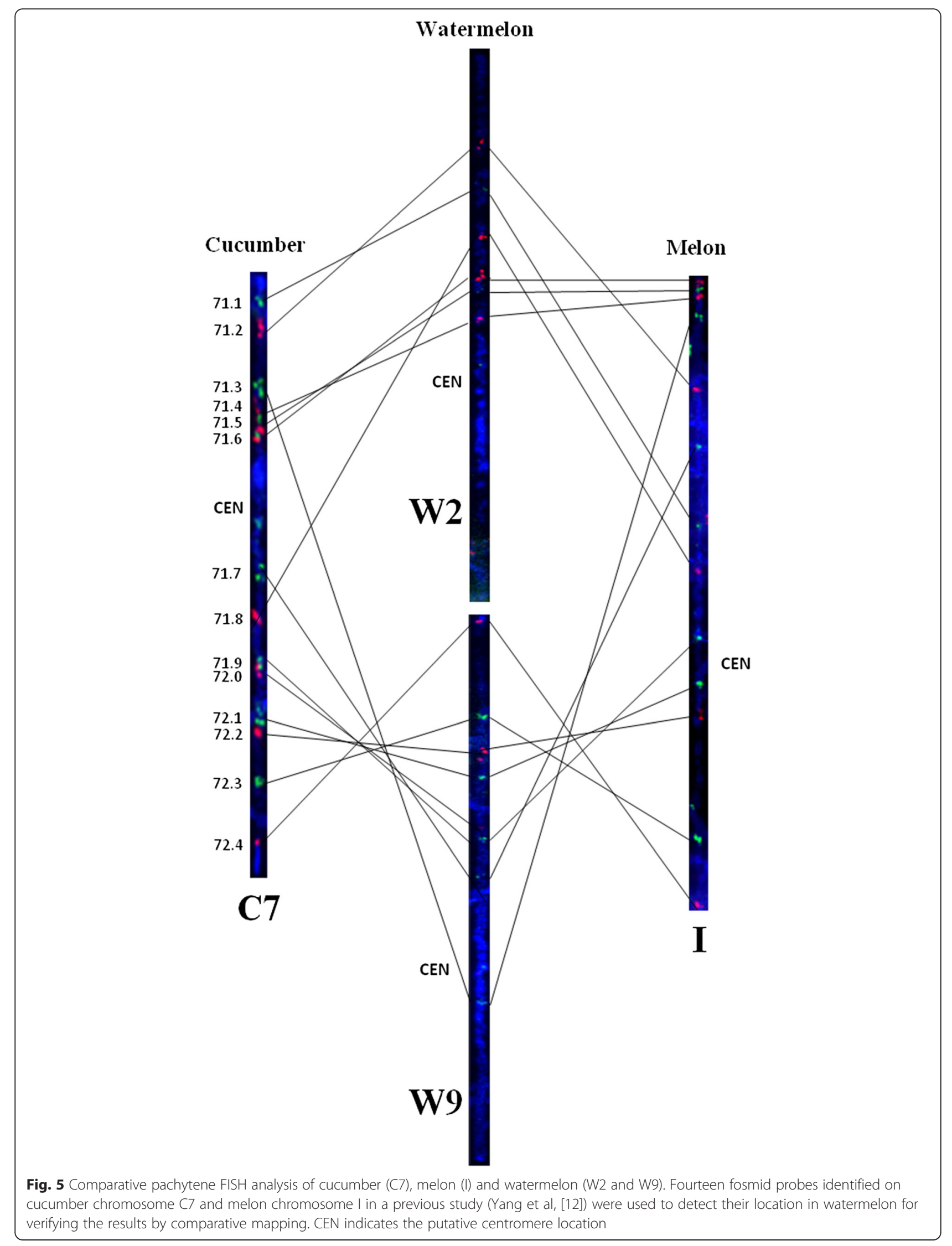


watermelon W2 were collinear with melon I, which were divided into two genomic regions in accordance with blocks WMB8 and WMB9. Three inversions were detected between watermelon W9 and melon I by the remaining fosmid probes, which were associated with the blocks WMB56, WMB 58 and WMB60. Thus, the results of FISH mapping were completely consistent with these finding revealed by comparative mapping using crossspecies SSR markers.

\section{Application of SSR marker in watermelon genetic diversity and population structure analysis}

One hundred ninety two SSR markers were selected from eleven watermelon chromosomes to test their polymorphism in six genotypes of cultivated watermelon from diverse geographical origins (Additional file 2: Table S8). Among them, 91 (47.40\%) were polymorphic among the six accessions, while almost half of them $(47.25 \%)$ had dinucleotide repeats, followed by tri- (17.58 \%) and tetra- $(8.79 \%)$. To identify and validate an appropriate set of SSR markers for characterizing $C$. lanatus germplasm collections, 32 highly informative SSR markers were selected with at least two SSR primers on each chromosome (Additional file 1: Figure S2). These markers were used for fingerprinting in a germplasm panel of 134 accessions (Additional file 2: Table S1) of Citrullus genus including C. colocynthis, C. lanatus var. lanatus, C. lanatus var. citorides. The 32 SSR markers detected a total of 151 alleles with an average of 4.72 alleles per marker. The number of observed alleles $(\mathrm{Na})$ ranged from two to eight, the observed heterozygosity $(\mathrm{Ho})$ from 0.03 to 0.47 , and Shannon's information index (I) from 0.52 to 1.41. The PIC (polymorphic information content) value for each locus ranged from 0.22 to 0.64 with an average of 0.46 (Additional file 2: Table S9).

To estimate the genetic diversity among these Citrullus accessions, we constructed a phylogenetic tree using the UPGMA method and two clusters were delineated. The first cluster contained all twelve C. lanatus var. citorides accessions and three C. colocynthis acessions (W2, W3 and W5).The second cluster contained the remaining two C. colocynthis acessions (W1 and W4) and all C. lanatus var. lanatus accessions (Fig. 6). The accessions collected from the same continent were not completely clustered in the same subclade indicating watermelon have occured different migrations and exchange between continents. Furthermore, we used a model-based approach for population structure analysis to analyze the germplasm panel of 134 accessions. According to distribution of $\Delta \mathrm{K}$ values, there was only one peak of $\Delta \mathrm{K}$ when $\mathrm{K}=2(\Delta \mathrm{K}=202.11$, Additional file 1: Figure S3) suggesting these 134 accessions were grouping into two populations (Fig. 7), which was almost completely consistent with the dendrogram. For example, W1 and W4 were two C. colocynthis acessions, but they were grouped with these $C$. lanatus var. lanatus accessions both in the genetic diversity and structure analysis. There were also three exceptions (W5, W9 and W12) which were clustered in group I in the dendrogram but sharing large admixed ancestry with $C$. lanatus var. lanatus population.

\section{Discussion}

Frequency, distribution and characterization of microsatellites in the watermelon genome

Discovery and mining of genomic SSR loci using whole genome sequences has had successful applications in a lot of plant species such as cucumber [23], cotton [34, 35], foxtail millet [24] and Brachypodium [36]. In the current study, a total of 39,523 microsatellites were identified from the watermelon genome assembly with a density of $111 \mathrm{SSR} / \mathrm{Mb}$. The number of microsatellites and their density identified in our study was lower than that in cucumber (552 SSR/Mb) and Arabidopsis (371 SSR/Mb) [23], maize (120 SSR/ $\mathrm{Mb}$ ) and wheat (163 SSR/Mb) [37]. One main reason for these differences may be due to variations in the search parameters used for detection of microsatellites. For example, different repeat types (mono- to pentanucleotides versus mono- to octanucleotides) of different minimum lengths (12 bp versus $18 \mathrm{bp}$ ) were searched using different software. In this study, we analyzed the distribution and frequency of microsatellites with motifs of 2-8 bp long and minimum lengths of $18 \mathrm{bp}$ or minimum of 3 repeat units in watermelon genome assembly. The criterion we used was based on the fact that polymorphism levels and mutation rate correlate positively with the number of repeat units [38], and therefore a higher polymorphic ratio is expected for these SSR markers developed in this study.

Frequency analysis of various nucleotide repeats in watermelon revealed that dinucleotide repeats were the most abundant SSRs followed by tri-, tetra-, penta-, hepta-, hexa- and octonucleotide repeats (Fig. 1 and Table 1). This was different with the trend in other species. For example, the tetranucleotide repeats were the most abundant in cucumber, Medicago truncatula, Populus trichocarpa and Vitis vinifera, and the trinucleotide repeats were the most abundant in Glycine max, Arabidopsis thaliana, Oryza sativa and Sorghum bicolor [23]. Overall, the AT-rich motifs such as AT and AAT were the predominant SSRs repeats types in each class in watermelon, representing $73.53 \%$ and $74.55 \%$ in dinucleotide repeats and trinucleotide repeats, respectively. Conversely, GC-rich repeat SSR motifs were very rare in all the nucleotides repeats. This result is consistent with other studies indicating that genomic SSRs with GC-rich repeats are rare in dicot species [39, 40]. 


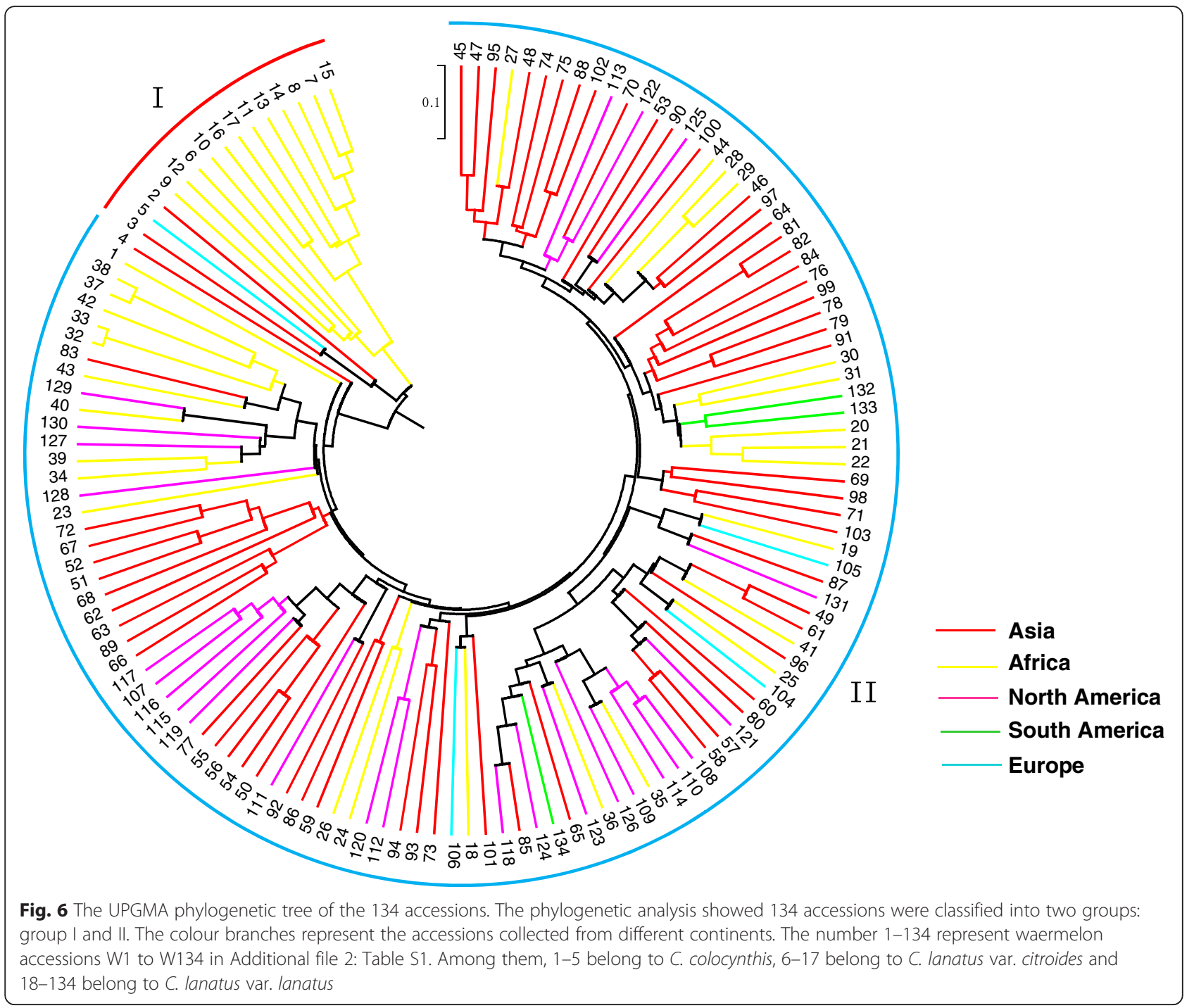

The frequency and distribution of different SSR type in different chromosomes revealed that the frequency of microsatellite loci was not positively correlated with the chromosome size in watermelon. While 3486 SSRs were detected from the $27.02 \mathrm{Mb}$ chromosome 6 , only 2456 microsatellite loci were detected on chromosome 4 which has a similar size of 24.32 Mb (Additional file 2: Table S2). One important explanation was that there were highly enriched repeat sequences on the short arm of chromosome 4 [7]. This could be further confirmed in the $24.26 \mathrm{Mb}$ unanchored scaffolds (chr0) on which only 889 microsatellite loci were detected. The remaining unassembled scaffolds from chr0 represented most of the repetitive fraction in watermelon genome. Our data is consistent with the observation in many plant species that SSR frequency is inversely to the proportion of repetitive DNA [41].
In silico PCR analysis of cross-species transferability of SSRs in watermelon

Since SSR markers possess significant specificity and show a high percentage of cross-species transferability, they have been used for various genotyping applications including studying cross-transferability and comparative genome mapping in related species [42-44]. In the present study, we tested the genome wide of crossspecies transferability of watermelon SSR markers in cucumber and melon by in silico PCR analysis. Among 32,869 SSRs examined, $832(2.53 \%)$ and 925 (2.81\%) had one non-redundant PCR product in cucumber and melon genome assembly, respectively. Although cucumber and melon have different chromosome numbers, they both belong to Cucumis genus sharing a common ancestor [33]. In our study, we found that the number of cross-species transferable SSR markers of watermelon was very close in cucumber and melon, which further 


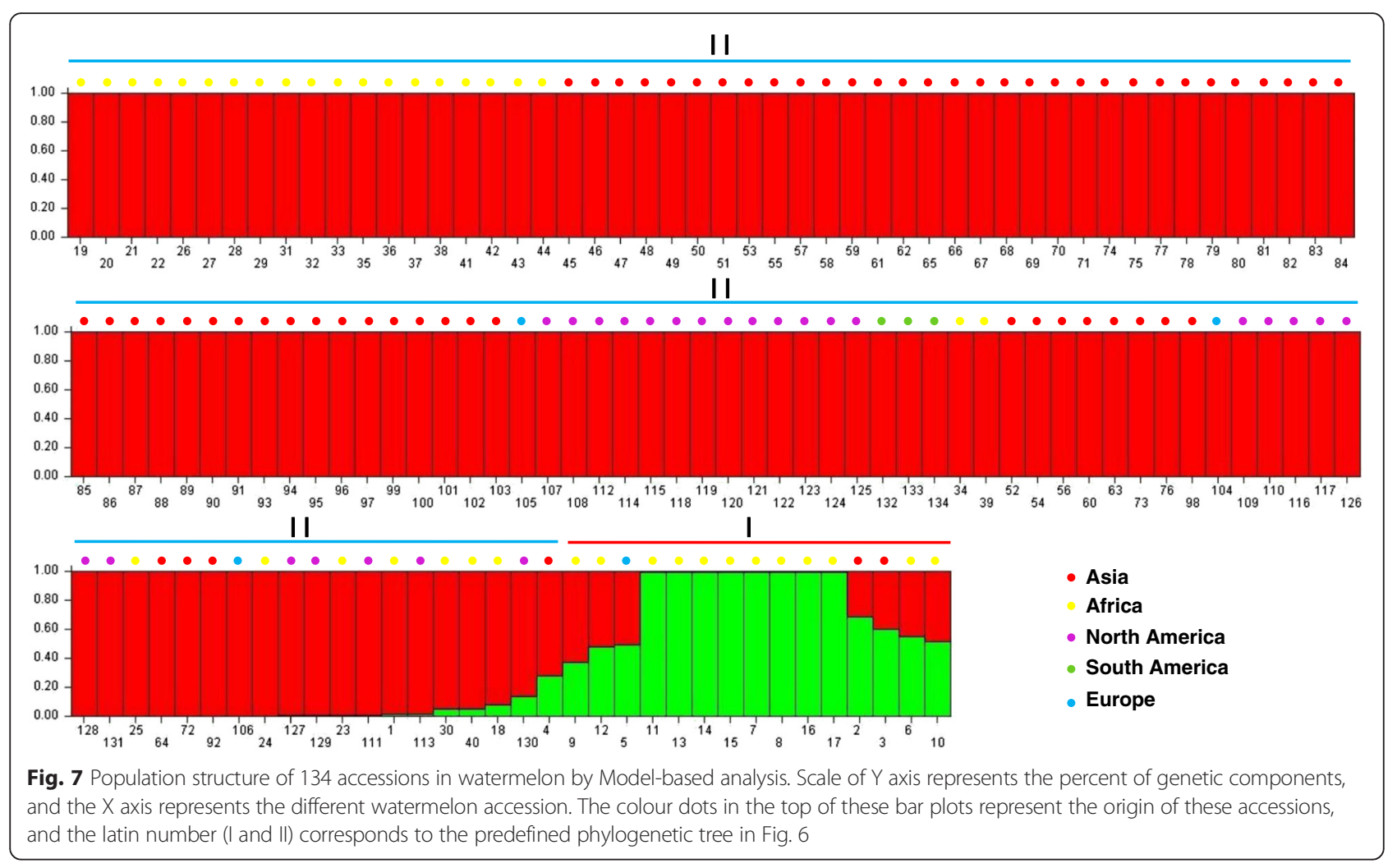

confirmed their evolutionary relationship that cucumber and melon were closely related, but distantly related with watermelon. It should be pointed out that the frequency of cross-species SSR markers in cucumber and melon was probably underestimated for two reasons. First, the incomplete genome assembly of cucumber and melon which represent only 53 and $83 \%$ of estimated cucumber and melon genome, respectively [27, 30]. Second, 59 and 44 SSR markers had multiply PCR products in cucumber and melon which were excluded for cross-species analysis. These cross-species transferable SSR markers covered 98.89 and $98.29 \%$ of the cucumber and melon genome assembly, respectively. It should be noted that these cross-species transferable SSR markers had high density in both ends of watermelon chromosomes and only few of them were located in centromere regions (Fig. 3) which usually contains high repetitive sequence varying from species [43, 45]. These crossspecies transferable SSR markers that generate one in silico PCR product should be the putative single-locus markers and could be especially useful in genetic map construction and gene mapping.

Furthermore, we investigated the frequency of different SSR motifs in 448 cross-three-species transferable SSR markers which were supposedly highly conserved. The frequency and distribution of microsatellite in these conserved 448 cross-species SSR markers were not consistent with that in the watermelon genome. For example, AG/CT was the most abundant motif in dinucleotides with a frequency of $59.12 \%$ in the 448 crossspecies SSR markers, while AT/TA (73.53 \%) was the predominant motif in the dinucleotides in the watermelon genome and AG/CT was only accounting for $22.10 \%$ of all dinucleotides (Additional file 1: Figure S1). In cucumber, AG/CT was the predominant dinucleotide in the EST-SSR [23] suggesting AG/CT was abundant in gene coding regions. This was confirmed by BLAST searching of cross-species transferable SSR markers in the CDS database of watermelon, and almost half of them were located in the coding regions. This might be because AG-rich regions are relatively stable, resulting in less replication slippage and usually distributed in exons, where polymorphisms occur less frequently [41]. Only 21 and 19 SSR markers were identified in watermelon EST and unigenes, respectively, which was probably due to the low coverage of EST collected in this database.

\section{Complicated syntenic pattern between watermelon withcucumber andmelon}

Syntenic relationship revealed by comparative mapping has been carried out in a number of economically important plant families including the Poaceae, Solanaceae, Brassicaceae and Rosaceae [44, 46-48]. The studies on chromosome relationship in the Cucurbitaceae family largely focused on the Cucumis genus to 
understand the mechanisms of dysploid chromosome reduction from $n=12$ to $n=7[12,27,29,31]$. In the present study, the large number of cross-species SSR markers provided a good opportunity to uncover the syntenic relationships among watermelon, melon and cucumber genomes at a high resolution level which enabled us to detect different patterns of chromosome evolution for three cucurbit species. We identified 84 and 81 watermelon-cucumber and watermelon-melon syntenic blocks, respectively. In most cases, each watermelon chromosome showed major synteny to at least three cucumber chromosomes and three melon chromosome, respectively (Table 3 and Fig. 3), indicating that these chromosomes have undergone chromosome fission after its divergence from the ancestor leading to cucumber and melon. Watermelon chromosome W3 and W10 had the simplest syntenic pattern to cucumber with each of them only corresponding to two cucumber chromosomes. For example, 86 of shared SSR markers on watermelon W10 were mapped on cucumber C3, and five SSR markers were syntenic to cucumber C4. In-depth analysis revealed the shared SSR markers were further divided into three collinear blocks (WCB68, 70 and 72) and three inverted blocks (WCB67, 69 and 73) suggesting the very complicated evolutionary dynamics of chromosomes evolution in the Cucurbitaceae family. Although watermelon $(n=11)$ and melon $(n=12)$ have similar chromosome number, the syntenic patterns were not simple one to one chromosome. Most watermelon chromosome were syntenic to three or four melon chromosomes, indicating the chromosome rearrangements between melon and watermelon were much more complicated compared with other closely related species with similar chromosome number in the same family. For example, in Rosaceae, most of peach $(n=8)$ chromosome showed major synteny to one strawberry $(n=7)$ chromosome [46]. This implies that much more structural changes between melon and watermelon have occurred during their karyotype evolution, speciation and local adaptation.

The evolutionary relationships among three cucurbit species were further analysed by investigating the syntenic blocks in watermelon, melon and cucumber. 25 syntenic blocks were identified that were shared by the three genomes (Additional file 2: Table S6), which covered about $57 \mathrm{Mb}$ representing $17.08 \%$ of watermelon genome assembly, suggesting these genomic regions may be highly conserved during chromosome evolution in the Cucurbitaceae family. Compared with these conserved blocks, other syntenic blocks were split into small blocks or rearranged into new blocks between three genomes. For example, the WCB6 on watermelon chromosome W1 (26.13-33.99 Mb) was collinear to cucumber chromosome $\mathrm{C6}$, which was divided into two block in melon (WMB5 and WMB6) (Additional file 2: Table S6). This phenomenon was further confirmed by comparative pachytene FISH on chromosome synteny of cucumber C7, melon I and watermelon W2 and W9. Previous studies have revealed that cucumber $\mathrm{C} 7$ was highly conserved with melon I and even in other farther related species of Cucumis genus, and mainly syntenic to watermelon chromosomes W2 and W9 [7, 12]. The large syntenic block WCB62 was an inversion between cucumber C7 and watermelon W9 confirmed by fosmid probes 71.7-72.4, while it was splite to several small inverted blocks in melon genome (Fig. 5 and Additional file 2: Table S3), indicating this genomic region have undergone extra structure changes in melon lineage after its divergence from the common ancestor of Cucumis. In addition, the syntenic blocks identified in this study will also help to improve the genome assembly. For example, only one markers ClSSR03519 in block WMB6 developed from scaffolds unanchored to chromosomes in melon, while 26 shared SSR markers in this block were highly continuously distributed on watermelon chromosome W1 and melon chromosome VIII, suggesting the scaffold from which CISSR03519 was developed should be anchored on melon chromosome VIII.

\section{The genetic diversity and population structure of watermelon}

Due to the scarcity of highly polymorphic, user-friendly molecular markers in watermelon, high-density genetic maps were not available until recently [8]. Most genetic diversity and linkage maps have been used low throughput, anonymous, dominant markers such as RAPDs, AFLPs and SRAPs in watermelon $[49,50]$, making them difficult in applications like map-based cloning, and maker-assisted selection. The large number of genome wide SSR markers developed from watermelon genome in this study should provide a valuable resource to the watermelon community in various marker-based studies such as genetic diversity analysis, gene mapping or cloning, genome wide association study (GWAS) and marker-assisted selection for watermelon breeding. By validating 192 SSR markers (Additional file 2: Table S8), as high as $47.40 \%$ of them were polymorphic in six accessions of $C$. lanatus var. lanatus, suggesting these SSR markers will be useful in genetic diversity study. Furthermore, the cross-species transferable SSR markers identified by in silico PCR analysis in cucumber and melon genome could also be applied in studies of development of practical markers in other closely related species of same genus.

In our study, 32 highly informative SSR markers were used for genetic diversity anlaysis and inferring population structure of 134 watermelon accessions revealed that they were divided into two groups in the UPGMA tree which 
were consistent with their population structure (Fig. 6 and Fig. 7). Interestingly, two C. colocynthis accessions W1 and W4 were clustered with all C. lanatus var. lanatus accessions in group II, and population structure also revealed that they shared a large part background with $C$. lanatus var. lanatus, suggesting this two $C$. colocynthis accessions are more closely related to C. lanatus var. lanatus (Fig. 7), which is especially true for the accession W1 (PI 388770). PI 388770 was also used previously by Levi et al. [6], who also found that this accession was closer to $C$. lanatus var. lanatus. Therefore these two accessions may merit additional investigations to resolve the anomaly. The structure analysis revealed that $\mathrm{K}=2$ was the best value for classfication of the 134 watermelon accessions which was different with other studies. Levi et al. [6] used high frequency oligonucleotides targeting active gene (HFO-TAG) markers grouping 96 watermelon accessions into four population, while Reddy et al. [9] grouped 96 different watermelon accessions into five population revealed by 201 SSR markers. This suggested the population structure of watermelon varied by using different accessions and different molecular marker numbers.

Watermelon has a very narrow genetic base and evaluating the phylogenetic relationships among different species in Citrullus genus will help us for better improving the watermelon cultivars and broaden its gene pool specially from the primary gene pools: $C$. lanatus var. lanatus, $C$. lanatus var. citorides and Citrullus colocynthis [2, 5, 51]. For example, three accessions (W8, W9 and W15) of $C$. lanatus var. citorides were previously reported resistant to anthracnose race 2 [52], one accession W11 was reported to contain resistance to Fusarium wilt [53] and another accession W1 of C. colocynthis showed fruit rot resistance [54]. These sources of resistance to different diseases will be valuable in watermelon breeding programs aimed at enhancing disease resistance. In addition, the large differentiation among populations indicates that each population may possess its own alleles and haplotypes. Therefore, crosses between different populations will broaden the genetic diversity within current breeding programs and may increase heterosis.

\section{Conclusions}

We developed genome wide microsatellite and characterized the frequency and distribution of different motifs in watermelon. The dinucleotides were the most abundant type and AT-rich motifs were predominant motifs in all nucleotide repeat in watermelon genome. Furthermore, the cross-species transferable SSR were detected in melon and cucumber by in silico PCR analysis, and these large number share SSR markers provide a higher level of resolution for comparative mapping to understand genomic relationships among these three species in the Cucurbitaceae family. Most of the chromosome in melon and cucumber were syntenic to three or four chromosomes of watermelon. The chromosome synteny suggested complicated structure rearrangements occurred from watermelon to melon and cucumber after their divergence from common ancestor. In addition, 32 high polymorphism SSR markers were used to study the genetic diversity of 134 watermelon accessions which were clustered into two groups. The large number SSR markers in watermelon and cross-species transferable SSR identified in this study could be applied in many research areas such as map construction, comparative mapping and marker-assisted trait selection, and also provide an important marker resource to other closely related and genome unsequenced species in Cucurbitaceae family.

\section{Methods \\ Plant material and DNA isolation}

A total of 134 watermelon Plant Introduction (PI) accessions from diverse geographic regions were selected for genetic diversity analysis (Additional file 2: Table S1). Of them, 117, 12 and 5 are designated as C. lanatus var. lanatus, C. lanatus var. citroides and C. colocynthis, respectively. Geographically, 62 were from Asian, 4 from Europe, 40 from Africa, 25 from North America and 3 from South America. Unexpanded young leaves from these accessions were collected into $2.0 \mathrm{~mL}$ microcentrifuge tubes, lyophilized in a freeze dryer, and ground into fine powder. Genomic DNA was extracted using the CTAB method [55].

\section{SSR identification and primer design}

The genome assembly of watermelon were downloaded from Cucurbit Genomics Database (http:// www.icugi.org/cgi-bin/ICuGI/index.cgi). To develop a higher polymorphism SSR primer set for future study, the criteria used for microsatellite identification in this study was from 2- to 8-bp motifs, and mononucleotides were not considered due to the difficulty of distinguishing bona fide microsatellites from sequencing or assembly error. DNA sequences were searched for both perfect and compound microsatellites, with a basic motif of 2-8 bp, using the computer program MISA (Microsatellite identification tool) [56]. Repeats with a minimum length of 18 (for di- to tetranucleotides), 20 (for pentanucleotides), 24 (for hexanucleotides), 21 (for heptanucleotides), and $24 \mathrm{bp}$ (for octanucleotides) were recorded. The physical positions of the SSRs found in the chromosomes were also recorded, and oligonucleotide primers were designed for the genomic sequence flanking these SSRs using Primer3 (v. 1.1.4) software [57]. Primers were designed to generate amplicons of 100-300 bp in length with the following minimum, optimum and maximum 
values for Primer3 parameters: primer length (bp): 18-20-24; $\mathrm{Tm}\left({ }^{\circ} \mathrm{C}\right)$ : 50-55-60. Other parameters used the default program values.

\section{In silico analysis of watermelon SSR markers in cucumber and melon genome}

Using an in silico PCR strategy, all the SSR primer developed from watermelon genome assembly were used to BLAST search in cucumber Gy14 [27] and melon DHL92 genome assembly [30] which were downloaded from http://wenglab.horticulture.wisc.edu/cucumber-genomedatabase/ and https://melonomics.net, respectively. This was performed with a custom Perl script that used the NCBI BLASTN program as a search engine with expect value of 10 and filtering. We allowed up to 5 nucleotide mismatches at the $5^{\prime}$ end of the primer but no mismatches at the 3 ' end, and a minimum of $90 \%$ overall match homology. The in silico PCR products containing single copy or multiple copies were both recorded for further analysis. Furthermore, the set of cross-three-species transferable SSR were selected by comparing common SSR markers between watermelon with melon and cucumber. To investigate the distribution of these highly conserved SSR markers in watermelon, the in silico PCR product sequences were used to blast search in EST, unigene and CDS database of watermelon from the cucurbit genomics database (http:// www.icugi.org) with a threshold E-10.

To establish the syntenic chromosomes relationships between watermelon with cucumber and melon, we only kept the SSR markers in melon and cucumber genomes which had single in silico PCR product. In addition, these shared SSR markers located on the chromosomal unanchored scaffolds were further filtered. Then the chromosome relationship among three species was inferred by the remaining shared SSR markers. The SSR marker-based syntenic relationships among cucumber, melon and watermelon were finally visualized with visualization blocks in Circos software v 0.55 (http://cir cos.ca) [58].

\section{PCR amplification and validation of selected SSRs}

One hundred and ninety-two SSR markers were selected to validate the polymorphism in six accessions of watermelon, and 32 of them with informative and unambiguous bands were further chosen in the genetic diversity study. Each polymerase chain reaction (PCR) contained $25 \mathrm{ng}$ template DNA, $0.5 \mu \mathrm{M}$ each of forward and reverse primers, $0.2 \mathrm{mM}$ dNTP mix, 0.5 unit of Taq DNA polymerase and $1 \times$ PCR buffer in a total volume of $10.0 \mu \mathrm{l}$. The amplification was carried out at initial denaturing step at $94{ }^{\circ} \mathrm{C}$ for 4 min followed by 30 cycles of $94{ }^{\circ} \mathrm{C}$ for $20 \mathrm{sec}, 55^{\circ} \mathrm{C}$ for $45 \mathrm{~s}$ and $72{ }^{\circ} \mathrm{C}$ for $1 \mathrm{~min}$. In the last cycle, primer extension was performed at $72{ }^{\circ} \mathrm{C}$ for $10 \mathrm{~min}$ and storage at $4{ }^{\circ} \mathrm{C}$ till electrophoresis. The PCR products were size-fractionated in a $9 \%$ polyacrylamide gel. The 100-bp DNA ladder was used as molecular size marker. After gel electrophoresis, band patterns were visualized with silver staining, and gel images were taken with a digital camera.

\section{Genetic diversity and population structure analysis}

The genomic DNA fragments from SSRs generated clear and unambiguous bands of various molecular weight sizes were scored for watermelon 134 accessions and calculated into co-dominant genotypic matrix in GeneAlEx 6.5 [59], then the UPGMA method was used to construct the dendrogram by software MEGA5 [60]. The observed (Na) and effective $(\mathrm{Ne})$ number of alleles, Shannon's information index (I), and levels of observed (Ho) and expected (He) heterozygosity were calculated by GeneAlEx 6.5. Polymorphic information content (PIC) for molecular markers was calculated as par the formula: $\mathrm{PIC}=\Sigma \mathrm{Pij}^{2}$ where Pij is the frequency of the $j^{\text {th }}$ pattern for marker $j$ and the summation extends over $\mathrm{n}$ patterns.

The model-based program STRUCTURE was used to infer population structure by the program STRUCTURE $2.3[61,62]$. The program was run with SSR markers for $\mathrm{k}$-values from 1 to 10 , and the number of populations (K) was determined using an admixture model with correlated alleles. Twenty independent runs of 100,000 Markov Chain Monte Carlo generations after 50,000 generation burn-in periods were used to estimate each value of $K$. The optimal $\mathrm{K}$ depends on the peak of $\Delta \mathrm{K}=$ mean $(|\operatorname{Ln} " \mathrm{P}(\mathrm{D})|) /(\operatorname{sdLnP}(\mathrm{D}))$, where $|\operatorname{Ln} " \mathrm{P}(\mathrm{D})|$ denotes the absolute value of the second order rate of change of $\operatorname{LnP}(\mathrm{D})$ and $\operatorname{sdLnP}(\mathrm{D})$ the standard deviation of the $\operatorname{LnP}(\mathrm{D})$. To infer the true $\mathrm{K}$, we run another twenty independent runs for the $\mathrm{K}$ from 1-5 with 750,000 Markov Chain Monte Carlo generations after 500,000 generation burn-in periods.

\section{Comparative fluorescence in situ hybridization (FISH)}

To examine and validate chromosome rearrangements between cucumber $\mathrm{C} 7$, melon I, watermelon W2 and W9, 14 fosmid probes identified on cucumber C7 [12] were used in comparative FISH mapping of meiotic pachytene chromosomes prepared from pollen mother cells of melon and watermelon. The physical order of adjacent fosmid clones in each chromosome was determined by two-color FISH. The FISH procedure was performed as described by Koo et al. [63]. Biotin- and digoxigenin-labeled probes were detected with Alexa Fluor 488 streptavidin antibody (Invitrogen, Carlsbad, CA) and rhodamine-conjugated anti-digoxigenin antibody (Roche Diagnostics USA, Indianapolis, IN), respectively. Chromosomes were counterstained by $4 \mathbb{C}$, 6-diamidino-2-phenylindole (DAPI) in 'Vector Shield' 
antifade solution (Vector Laboratories, Burlingame, CA). FISH signals were captured using a CCD camera. The images were processed using Meta Imaging Series 7.5 software (Molecular Devices, Downingtown, PA, USA).

\section{Additional files}

Additional file 1: Figure S1. The motif distribution of dinucleotide (A) trinucleotide (B) and tetranucleotide (C) in watermelon. Figure S2. The chromosome position of the 32 SSR markers in watermelon. Figure S3. Delta $\mathrm{K}$ distribution across various clusters $(\mathrm{K})$ as estimated by Structure Harvester. (PDF $833 \mathrm{~kb}$ )

Additional file 2: Table S1. Watermelon Plant introduction accessions (PIs) used in the present study. Table S2. Genome wide SSR primers developed in the watermelon genome. Table S3. List of cross-species SSR markers between watermelon and cucumber identified by in silico PCR. Table S4. List of crossspecies SSR markers between watermelon and melon identified by in silico PCR. Table S5. List of conserved SSR markers among watermelon, melon and cucumber genomes. Table S6. Syntenic blocks shared for cucumber, melon and watermelon genomes. Table 57. Information of fosmid clones used in fluorescence in situ hybridization (FISH). Table S8. List of 192 SSR primers used for polymorphism screening in watermelon. Table S9. Statistic of observed $(\mathrm{Na})$ and effective number of alleles $(\mathrm{Ne})$, observed $(\mathrm{Ho})$ and expected heterozygosity (He), polymorphic information content (PIC) and Shannon's information index (I) for 32 SSR markers. (XLSX 3673 kb)

\section{Abbreviations}

AFLP, amplified fragment length polymorphism; BLAST, basic local alignment search tool; CDS, coding sequence; EST, expressed sequence tag; FISH, fluorescence in situ hybridization; GWAS, genome wide association study; HFOTAG, high frequency oligonucleotides targeting active gene; MAS, markerassisted selection; Mb, million base pairs; MISA, microsatellite identification tool; NGS, next-generation sequencing; PCR, polymerase chain reaction; PI, plant introductions; PIC, polymorphic information content; QTL, quantitative trait loci; RAPD, random amplified polymorphic DNA; SNP, single nucleotide polymorphism; SRAP, sequence related amplified polymorphism; SSR, simple sequence repeats; WGS, whole-genome sequences

\section{Acknowledgements}

We thank professor Yufeng Wu of Nanjing Agricultural University in using Circos software. We are also grateful to the editor and reviewers for critically evaluating the manuscript and providing constructive comments for its improvement.

\section{Funding}

This research was supported by Scientific Innovation Fund of Henan Agricultural University (KJCX2015A11) and The Initial Funding for the introduction of talents of Henan Agricultural University (30600860).

\section{Availability of data and materials}

The detail information of genome-wide SSR markers developed in this study is provided in Additional file 2: Table S2. Furthermore, the watermelon materials used in this study are free to public, and anyone can require for the materials from college of Horticulture at Henan Agricultural University.

\section{Authors' contributions}

$\mathrm{HZ}$ performed the data analysis and wrote the manuscript. PS conducted validation of SSR polymorphism, genetic diversity and population structure analysis. KD performed the comparative FISH experiment. LG, YL and SS participated in SSR fingerprinting and genetic diversity analysis. LY and YW conceived the study, designed the experiments, wrote and revised the manuscript. All authors read and approved the final manuscript.

\section{Competing interests}

The authors declare that they have no competing interests.

\section{Consent for publication}

Not Applicable.
Ethics approval and consent to participate

Not Applicable.

\section{Author details}

${ }^{1}$ College of Horticulture, Henan Agricultural University, 63 Nongye Road, Zhengzhou 450002, China. ${ }^{2}$ Horticulture Department, University of Wisconsin, Madison, WI 53706, USA. ${ }^{3}$ US Department of Agriculture/Agricultural Research Service, Vegetable Crops Research Unit, 1575 Linden Drive, Madison, WI 53706, USA.

Received: 22 February 2016 Accepted: 1 July 2016

Published online: 05 August 2016

\section{References}

1. Levi A, Thomas C, Keinath A, Wehner T. Genetic diversity among watermelon (Citrullus lanatus and Citrullus colocynthis) accessions. Genet Resour Crop Evol. 2001;48(6):559-66.

2. Jarret RL, Merrick LC, Holms T, Evans J, Aradhya MK. Simple sequence repeats in watermelon (Citrullus lanatus (Thunb.) Matsum. \& Nakai). Genome. 1997;40(4):433-41.

3. Paris HS. Origin and emergence of the sweet dessert watermelon. Citrullus Lanatus Ann Bot-London. 2015;116(2):133-48.

4. Chomicki G, Renner SS. Watermelon origin solved with molecular phylogenetics including Linnaean material: another example of museomics. New Phytol. 2015;205(2):526-32.

5. Zamir D, Navot N, Rudich J. Enzyme polymorphism in Citrullus lanatus and C. colocynthis in Israel and Sinai. Plant Syst Evol. 1984;146(3-4):163-70.

6. Levi A, Thies JA, Wechter WP, Harrison HF, Simmons AM, Reddy UK, Nimmakayala P, Fei ZJ. High frequency oligonucleotides: targeting active gene (HFO-TAG) markers revealed wide genetic diversity among Citrullus spp. accessions useful for enhancing disease or pest resistance in watermelon cultivars. Genet Resour Crop Evol. 2013;60(2):427-40.

7. Guo S, Zhang J, Sun H, Salse J, Lucas WJ, Zhang H, Zheng Y, Mao L, Ren Y, Wang $Z$, et al. The draft genome of watermelon (Citrullus lanatus) and resequencing of 20 diverse accessions. Nat Genet. 2013;45(1):51-8.

8. Ren Y, Zhao H, Kou Q, Jiang J, Guo S, Zhang H, Hou W, Zou X, Sun H, Gong $G$, et al. A high resolution genetic map anchoring scaffolds of the sequenced watermelon genome. PLoS One. 2012;7(1):e29453.

9. Reddy UK, Abburi L, Abburi VL, Saminathan T, Cantrell R, Vajja VG, Reddy R, Tomason YR, Levi A, Wehner TC, et al. A genome-wide scan of selective sweeps and association mapping of fruit traits using microsatellite markers in watermelon. J Hered. 2015;106(2):166-76.

10. Lambel S, Lanini B, Vivoda E, Fauve J, Patrick Wechter W, Harris-Shultz KR, Massey L, Levi A. A major QTL associated with Fusarium oxysporum race 1 resistance identified in genetic populations derived from closely related watermelon lines using selective genotyping and genotyping-bysequencing for SNP discovery. Theor Appl Genet. 2014;127(10):2105-15.

11. Ren Y, Jiao D, Gong GY, Zhang HY, Guo SG, Zhang J, Xu Y. Genetic analysis and chromosome mapping of resistance to Fusarium oxysporum $\mathrm{f}$. sp niveum (FON) race 1 and race 2 in watermelon (Citrullus lanatus L.). Mol Breeding. 2015;35:183

12. Yang L, Koo DH, Li D, Zhang T, Jiang J, Luan F, Renner SS, Henaff E, Sanseverino W, Garcia-Mas J, et al. Next-generation sequencing, FISH mapping and synteny-based modeling reveal mechanisms of decreasing dysploidy in Cucumis. Plant J. 2014;77(1):16-30.

13. Bruford MW, Wayne RK. Microsatellites and their application to population genetic studies. Curr Opin Genet Dev. 1993;3(6):939-43.

14. Bo K, Ma Z, Chen J, Weng Y. Molecular mapping reveals structural rearrangements and quantitative trait loci underlying traits with local adaptation in semi-wild Xishuangbanna cucumber (Cucumis sativus L. var. xishuangbannanesis Qi et Yuan). Theor Appl Genet. 2015;128(1):25-39.

15. Li Y, Wen C, Weng Y. Fine mapping of the pleiotropic locus B for black spine and orange mature fruit color in cucumber identifies a $50 \mathrm{~kb}$ region containing a R2R3-MYB transcription factor. Theor Appl Genet. 2013;126(8):2187-96.

16. Lv J, Qi J, Shi Q, Shen D, Zhang S, Shao G, Li H, Sun Z, Weng Y, Shang Y, et al. Genetic diversity and population structure of cucumber (Cucumis sativus L. PLoS One. 2012;7(10):e46919.

17. Garke C, Ytournel F, Bed'hom B, Gut I, Lathrop M, Weigend S, Simianer H. Comparison of SNPs and microsatellites for assessing the genetic structure of chicken populations. Anim Genet. 2012;43(4):419-28. 
18. Hamblin MT, Warburton ML, Buckler ES. Empirical comparison of Simple Sequence Repeats and single nucleotide polymorphisms in assessment of maize diversity and relatedness. PLoS One. 2007;2(12):e1367.

19. Huang D, Zhang $Y$, Jin M, Li H, Song Z, Wang Y, Chen J. Characterization and high cross-species transferability of microsatellite markers from the floral transcriptome of Aspidistra saxicola (Asparagaceae). Mol Ecol Resour. 2014;14(3):569-77

20. Singh RK, Jena SN, Khan S, Yadav S, Banarjee N, Raghuvanshi S, Bhardwaj V, Dattamajumder SK, Kapur R, Solomon S, et al. Development, cross-species/genera transferability of novel EST-SSR markers and their utility in revealing population structure and genetic diversity in sugarcane. Gene. 2013;524(2):309-29.

21. Cota LG, Moreira PA, Menezes EV, Gomes AS, Ericsson AR, Oliveira DA, Melo Jr AF. Transferability and characterization of simple sequence repeat markers from Anacardium occidentale to A. humile (Anacardiaceae). Genet Mol Res. 2012;11(4):4609-16.

22. Wang YW, Samuels TD, Wu YQ. Development of 1,030 genomic SSR markers in switchgrass. Theor Appl Genet. 2011;122(4):677-86.

23. Cavagnaro PF, Senalik DA, Yang L, Simon PW, Harkins $\Pi$, Kodira CD, Huang $\mathrm{S}$, Weng Y. Genome-wide characterization of simple sequence repeats in cucumber (Cucumis sativus L.). BMC Genomics. 2010;11:569.

24. Zhang S, Tang CJ, Zhao Q, Li J, Yang LF, Qie LF, Fan XK, Li L, Zhang N, Zhao $M C$, et al. Development of highly polymorphic simple sequence repeat markers using genome-wide microsatellite variant analysis in Foxtail millet [Setaria italica (L.) P. Beauv.]. BMC Genomics. 2014;15:78.

25. Shi J, Huang S, Zhan J, Yu J, Wang X, Hua W, Liu S, Liu G, Wang H. Genomewide microsatellite characterization and marker development in the sequenced Brassica crop species. DNA Res. 2014;21(1):53-68.

26. Sarika, Arora V, Iquebal MA, Rai A, Kumar D. In silico mining of putative microsatellite markers from whole genome sequence of water buffalo (Bubalus bubalis) and development of first BuffSatDB. BMC Genomics. 2013;14:43.

27. Yang L, Koo D-H, Li Y, Zhang X, Luan F, Havey MJ, Jiang J, Weng Y. Chromosome rearrangements during domestication of cucumber as revealed by high-density genetic mapping and draft genome assembly. Plant J. 2012;71(6):895-906

28. Zhang HY, Wang H, Guo SG, Ren Y, Gong GY, Weng YQ, Xu Y. Identification and validation of a core set of microsatellite markers for genetic diversity analysis in watermelon, Citrullus lanatus Thunb. Matsum Nakai Euphytica. 2012;186(2):329-42.

29. Huang S, Li R, Zhang Z, Li L, Gu X, Fan W, Lucas WJ, Wang X, Xie B, Ni $P$, et al. The genome of the cucumber, Cucumis sativus L. Nat Genet. 2009;41(12):1275-81.

30. Garcia-Mas J, Benjak A, Sanseverino W, Bourgeois M, Mir G, Gonzalez VM, Henaff E, Camara F, Cozzuto L, Lowy E, et al. The genome of melon (Cucumis melo L.). Proc Natl Acad Sci U S A. 2012;109(29):11872-7.

31. Li DW, Cuevas HE, Yang LM, Li YH, Garcia-Mas J, Zalapa J, Staub JE, Luan FS, Reddy U, He XM, et al. Syntenic relationships between cucumber (Cucumis sativus L.) and melon (C. melo L.) chromosomes as revealed by comparative genetic mapping. BMC Genomics. 2011;12:396.

32. Schaefer $\mathrm{H}$, Heibl C, Renner SS. Gourds afloat: a dated phylogeny reveals an Asian origin of the gourd family (Cucurbitaceae) and numerous oversea dispersal events. Proc Biological Sci Royal Soc. 2009;276(1658):843-51.

33. Ghebretinsae AG, Thulin M, Barber JC. Relationships of cucumbers and melons unraveled: Molecular phylogenetics of Cucumis and related genera (Benincaseae, Cucurbitaceae). Am J Bot. 2007;94(7):1256-66.

34. Lu C, Zou C, Zhang Y, Yu D, Cheng H, Jiang P, Yang W, Wang Q, Feng $X$, Prosper MA, et al. Development of chromosome-specific markers with high polymorphism for allotetraploid cotton based on genomewide characterization of simple sequence repeats in diploid cottons (Gossypium arboreum L. and Gossypium raimondii Ulbrich). BMC Genomics. 2015;16:55

35. Wang Q, Fang L, Chen J, Hu Y, Si Z, Wang S, Chang L, Guo W, Zhang T. Genome-wide mining, characterization, and development of microsatellite markers in gossypium species. Sci Rep. 2015;5:10638.

36. Sonah H, Deshmukh RK, Sharma A, Singh VP, Gupta DK, Gacche RN, Rana JC, Singh NK, Sharma TR. Genome-wide distribution and organization of microsatellites in plants: an insight into marker development in Brachypodium. PLoS One. 2011;6(6):e21298.

37. Huo N, Lazo GR, Vogel JP, You FM, Ma Y, Hayden DM, Coleman-Derr D, Hill TA, Dvorak J, Anderson OD, et al. The nuclear genome of Brachypodium distachyon: analysis of BAC end sequences. Funct Integr Genomics. 2008;8(2):135-47.

38. Weber JL. Informativeness of human (dC-dA)n. (dG-dT)n polymorphisms. Genomics. 1990;7(4):524-30.

39. Wang Z, Weber JL, Zhong G, Tanksley SD. Survey of plant short tandem DNA repeats. Theor Appl Genet. 1994;88(1):1-6.

40. Tangphatsornruang S, Somta P, Uthaipaisanwong P, Chanprasert J, Sangsrakru D, Seehalak W, Sommanas W, Tragoonrung S, Srinives P. Characterization of microsatellites and gene contents from genome shotgun sequences of mungbean (Vigna radiata (L.) Wilczek). BMC Plant Biol. 2009;9:137.

41. Morgante M, Hanafey M, Powell W. Microsatellites are preferentially associated with nonrepetitive DNA in plant genomes. Nat Genet. 2002;30(2):194-200.

42. Kumari K, Muthamilarasan M, Misra G, Gupta S, Subramanian A, Parida SK, Chattopadhyay D, Prasad M. Development of eSSR-Markers in and Their Applicability in Studying Genetic Diversity, Cross-Transferability and Comparative Mapping in Millet and Non-Millet Species. PLoS One. 2013:8(6):e67742.

43. Mathithumilan B, Kadam NN, Biradar J, Reddy SH, Ankaiah M, Narayanan MJ, Makarla U, Khurana P, Sreeman SM. Development and characterization of microsatellite markers for Morus spp. and assessment of their transferability to other closely related species. BMC Plant Biol. 2013;13:194.

44. Lim GA, Jewell EG, Li X, Erwin TA, Love C, Batley J, Spangenberg G, Edwards D. A comparative map viewer integrating genetic maps for Brassica and Arabidopsis. BMC Plant Biol. 2007;7:40

45. Kamm A, Galasso I, Schmidt T, Heslop-Harrison JS. Analysis of a repetitive DNA family from Arabidopsis arenosa and relationships between Arabidopsis species. Plant Mol Biol. 1995;27(5):853-62.

46. Jung S, Cestaro A, Troggio M, Main D, Zheng P, Cho I, Folta KM, Sosinski B, Abbott A, Celton JM, et al. Whole genome comparisons of Fragaria, Prunus and Malus reveal different modes of evolution between Rosaceous subfamilies. BMC Genomics. 2012;13:129.

47. Doganlar S, Frary A, Daunay MC, Lester RN, Tanksley SD. A comparative genetic linkage map of eggplant (Solanum melongena) and its implications for genome evolution in the solanaceae. Genetics. 2002;161(4):1697-711.

48. Luo MC, Deal KR, Akhunov ED, Akhunova AR, Anderson OD, Anderson JA, Blake N, Clegg MT, Coleman-Derr D, Conley EJ, et al. Genome comparisons reveal a dominant mechanism of chromosome number reduction in grasses and accelerated genome evolution in Triticeae. Proc Natl Acad Sci U S A. 2009:106(37):15780-5.

49. Solmaz I, Sari N, Aka-Kacar Y, Yalcin-Mendi NY. The genetic characterization of Turkish watermelon (Citrullus lanatus) accessions using RAPD markers. Genet Resour Crop Evol. 2010;57(5):763-71.

50. Hashizume T, Shimamoto I, Hirai M. Construction of a linkage map and QTL analysis of horticultural traits for watermelon [Citrullus lanatus (THUNB.) MATSUM \& NAKAI] using RAPD, RFLP and ISSR markers. Theor Appl Genet. 2003;106(5):779-85.

51. Thies JA, Ariss JJ, Hassell RL, Olson S, Kousik CS, Levi A. Grafting for Management of Southern Root-Knot Nematode, Meloidogyne incognita, in Watermelon. Plant Dis. 2010:94(10):1195-9.

52. Sowell Jr G, Rhodes BB, Norton JD. New sources of resistance to watermelon anthracnose. J Amer Soc Hort Sci. 1980;105:197-9.

53. Netzer D, Martyn RD. PI 296341, a source of resistance in watermelon to race 2 of Fusarium oxysporum f.sp. niveum. Plant Dis. 1989;73:518.

54. Kousik CS, Ikerd JL, Wechter P, Harrison H, Levi A. Resistance to Phytophthora Fruit Rot of Watermelon Caused by Phytophthora capsici in U. S. Plant Introductions. HortSci. 2012;47(12):1682-9.

55. Murray MG, Thompson WF. Rapid isolation of high molecular weight plant DNA. Nucleic Acids Res. 1980;8(19):4321-5.

56. Thiel T, Michalek W, Varshney RK, Graner A. Exploiting EST databases for the development and characterization of gene-derived SSR-markers in barley (Hordeum vulgare L.). Theor Appl Genet. 2003;106(3):411-22.

57. Rozen S, Skaletsky H. Primer3 on the WWW for general users and for biologist programmers. Methods Mol Biol. 2000;132:365-86.

58. Krzywinski M, Schein J, Birol I, Connors J, Gascoyne R, Horsman D, Jones SJ, Marra MA. Circos: An information aesthetic for comparative genomics. Genome Res. 2009:19(9):1639-45.

59. Peakall R, Smouse PE. GenAlEx 6.5: genetic analysis in Excel. Population genetic software for teaching and research-an update. Bioinformatics. 2012;28(19):2537-9. 
60. Tamura K, Peterson D, Peterson N, Stecher G, Nei M, Kumar S. MEGA5: Molecular Evolutionary Genetics Analysis Using Maximum Likelihood, Evolutionary Distance, and Maximum Parsimony Methods. Mol Biol Evol. 2011;28(10):2731-9.

61. Pritchard JK, Stephens M, Donnelly P. Inference of population structure using multilocus genotype data. Genetics. 2000;155(2):945-59.

62. Evanno G, Regnaut S, Goudet J. Detecting the number of clusters of individuals using the software STRUCTURE: a simulation study. Mol Ecol. 2005;14(8):2611-20.

63. Koo DH, Nam YW, Choi D, Bang JW, de Jong H, Hur Y. Molecular cytogenetic mapping of Cucumis sativus and C. melo using highly repetitive DNA sequences. Chromosome Res. 2010;18(3):325-36.

Submit your next manuscript to BioMed Central and we will help you at every step:

- We accept pre-submission inquiries

- Our selector tool helps you to find the most relevant journal

- We provide round the clock customer support

- Convenient online submission

- Thorough peer review

- Inclusion in PubMed and all major indexing services

- Maximum visibility for your research

Submit your manuscript at www.biomedcentral.com/submit
Biomed Central 